\title{
Treatment of heparin-induced thrombocytopenia after cardiac surgery: Preliminary experience with fondaparinux
}

\author{
Federico Pappalardo, MD, a, Annamara Scandroglio, MD, ${ }^{\mathrm{a}}$ Giulia Maj, MD, ${ }^{\mathrm{a}}$ Alberto Zangrillo, MD, ${ }^{\mathrm{a}}$ and \\ Armando D'Angelo, MD, ${ }^{\mathrm{b}}$ Milan, Italy
}

Heparin-induced thrombocytopenia (HIT) is an immunemediated prothrombotic disorder that is often encountered after cardiac surgery. The appropriate alternative anticoagulant to be used in this setting is not univocal, especially for the coexistence of renal failure and high bleeding risk.

Fondaparinux is a factor Xa inhibitor via the action of antithrombin but devoid of antifactor II (thrombin) activity. It is modeled after a critical heparin pentasaccharide sequence; although the drug is identical in structure to the pentasaccharide domain found in heparin, it is too small to be recognized by the majority of heparin-reactive antibodies. ${ }^{1}$ We describe our early experience with fondaparinux in the management of HIT in critically ill patients after cardiac surgery.

\section{CLINICAL SUMMARY}

This retrospective cohort study was conducted on all patients diagnosed with HIT at our tertiary care, university cardiothoracic intensive care unit from January 2005 to December 2007. The diagnosis of HIT was based on clinicopathologic findings according to Warkentin and Greinacher. ${ }^{2}$

Fondaparinux was started after discontinuation of unfractionated or low-molecular-weight heparin; warfarin was overlapped when the platelet count increased to more than $100 \times 10^{9} / \mathrm{L}$. The dosage of fondaparinux was defined according to creatinine clearance $(\mathrm{CrCl})$ as calculated with the Cockcroft-Gault formula: $7.5 \mathrm{mg} / 24 \mathrm{~h}$ for $\mathrm{CrCl}$ more than $60 \mathrm{~mL} / \mathrm{min}, 5 \mathrm{mg} / 24 \mathrm{~h}$ for $\mathrm{CrCl}$ between 30 and 60 $\mathrm{mL} / \mathrm{min}$, and $2.5 \mathrm{mg} / 24 \mathrm{~h}$ for $\mathrm{CrCl}$ less than $30 \mathrm{~mL} / \mathrm{min}$.

\section{RESULTS}

During the study period, HIT was diagnosed in 18 patients (1.4\% of the overall cardiac surgical population); 11 patients received fondaparinux, and 8 patients received bivalir-

\footnotetext{
From the Department of Cardiovascular Anesthesia and Intensive Care, ${ }^{\mathrm{a}}$ and Coagulation Service and Thrombosis Research Unit, ${ }^{\mathrm{b}}$ IRCCS San Raffaele Hospital, Milan, Italy.

Disclosures: None.

Received for publication Feb 22, 2008; accepted for publication Nov 16, 2008; available ahead of print Feb 6, 2009.

Address for reprints: Federico Pappalardo, MD, Department of Cardiovascular Anesthesia and Intensive Care, IRCCS San Raffaele Hospital, Via Olgettina, 60, 20132 Milan, Italy (E-mail: pappalardo.federico@hsr.it).

J Thorac Cardiovasc Surg 2010;139:790-2

$0022-5223 / \$ 36.00$

Copyright (c) 2010 by The American Association for Thoracic Surgery doi:10.1016/j.jtcvs.2008.11.032
}

udin. The choice for fondaparinux was based on clinical basis: The most critically ill patients (ventilated, on hemofiltration, recent surgery) were treated with bivalirudin. Patient population data are presented in Tables 1 and 2.

The patients in the study group had a nadir platelet count at the time of diagnosis between 8 and $72 \times 10^{9} / \mathrm{L}$; the time lag from surgery was 6 to 40 days. All patients had a prompt increase of platelet count and decrease of HIT antibody titer. The duration of treatment was 4 to 17 days.

During fondaparinux therapy, 3 episodes of major bleeding (defined as life or organ threatening, requiring surgery or $>3$ units of packed red blood cells) were noted. Major bleeding occurred in 3 patients with a $\mathrm{CrCl}$ less than 30 $\mathrm{mL} / \mathrm{min}$ after low-risk procedures (epistaxis secondary to placement of a nasojejunal tube, stomal bleeding after tracheostomy, and hemothorax). They were treated by drug discontinuation, transfusions. and surgical hemostasis. One patient with a $\mathrm{CrCl}$ greater than $30 \mathrm{~mL} / \mathrm{min}$ had supratherapeutic anti-Xa activity without any apparent bleeding. No thromboembolic complications were recorded after initiation of therapy.

\section{DISCUSSION}

Because patients with HIT are at increased risk for thrombosis, anticoagulation is warranted. Multiple options for anticoagulation therapy are now available, placing an additional responsibility on health care workers for choosing the optimal therapy for each patient. The synthetic pentasaccharide fondaparinux binds to antithrombin and potentiates its inhibition of factor Xa. Anecdotal evidence suggests that fondaparinux may eventually prove to be valuable for preventing and treating thrombosis in patients with HIT. Furthermore, various small-scale in vitro studies have demonstrated a lack of cross-reactivity between fondaparinux and HIT antibodies. ${ }^{1}$ Multiple considerations need to be addressed when choosing an anticoagulant in the patients undergoing cardiac surgery.

The data reported suggest the following:

- On the basis of the theory of how coagulation factors interact, the results of in vitro studies, and the clinical outcomes, there is accumulating evidence that FXa may represent a better target for inhibition than FIIa. This is based on an understanding of the amplified nature of coagulation factor interactions and fibrin formation, the need for smaller doses of an anticoagulant to block coagulation 
TABLE 1. Patient characteristics

\begin{tabular}{|c|c|c|c|c|c|c|}
\hline Patient & Surgery & Heparin treatment (d) & Lowest platelet count & HIT Ab OD & SRA $(\%)$ & Days from surgery \\
\hline n 1 & $\mathrm{CABG}+\mathrm{MV}$ repair $+\mathrm{RF}$ & 40 & 72,000 & 1.8 & NA & 40 \\
\hline n 2 & AVR & 5 & 52,000 & 2 & NA & 8 \\
\hline n 3 & $\mathrm{MVR}+\mathrm{TV}$ repair $+\mathrm{RF}$ & 6 & 48,000 & 2 & NA & 8 \\
\hline n 4 & $\begin{array}{l}\text { AVR }+ \text { ascending aorta } \\
\text { replacement }\end{array}$ & 6 & 11,000 & 1.5 & NA & 7 \\
\hline n 5 & $\mathrm{MVR}+\mathrm{TVR}+\mathrm{ASD}$ closure & 10 & 61,000 & 0.68 & NA & 12 \\
\hline n 6 & $\mathrm{AVR}+\mathrm{MVR}$ & 5 & 8000 & NA & 100 & 6 \\
\hline n 7 & $\mathrm{AVR}+\mathrm{MVR}$ & 7 & 60,000 & 1.1 & NA & 7 \\
\hline n 8 & $\mathrm{MVR}+\mathrm{TV}$ repair $+\mathrm{CABG}$ & 6 & 37,000 & 1.9 & NA & 6 \\
\hline n 9 & MV repair & 7 & 61,000 & NA & NA & 11 \\
\hline n 10 & TAAA & 5 & 18,000 & 1.0 & NA & 7 \\
\hline n 11 & CABG & 5 & 13,000 & 1.5 & NA & 6 \\
\hline
\end{tabular}

HIT Ab OD, Heparin-induced thrombocytopenia antibody optical density; SRA, serotonin release assay; $N A$, not available; $M V$, mitral valve; $R F$, radiofrequency; $A V R$, aortic valve replacement; $M V R$, mitral valve replacement; $T V$, tricuspid valve; $T V R$, tricuspid valve replacement; $A S D$, atrial septal defect; $C A B G$, coronary artery bypass grafting; $T A A A$, thoracoabdominal aortic aneurysm.

TABLE 2. Clinical results

\begin{tabular}{|c|c|c|c|c|c|}
\hline Patient No. & Creatinine clearance & Duration of treatment $(d)$ & Bleeding & Thrombosis & Outcome \\
\hline 1 & $<30 \mathrm{~mL} / \mathrm{min} \mathrm{CVVH}$ & 4 & Epistaxis & No & Death \\
\hline 2 & $<30 \mathrm{~mL} / \mathrm{min}$ & 8 & No & No & Death \\
\hline 3 & $55 \mathrm{~mL} / \mathrm{min}$ & 7 & No & No & Discharged \\
\hline 4 & $<30 \mathrm{~mL} / \mathrm{min} \mathrm{CVVH}$ & 4 & Bleeding from tracheostomy & No & Death \\
\hline 5 & $>60 \mathrm{~mL} / \mathrm{min}$ & 7 & No & No & Discharged \\
\hline 6 & $>30 \mathrm{~mL} / \mathrm{min}$ & 6 & No & $\begin{array}{c}\text { Bilateral iliac and } \\
\text { femoral DVT }\end{array}$ & Discharged \\
\hline 7 & $45 \mathrm{~mL} / \mathrm{min}$ & 6 & No & No & Discharged \\
\hline 8 & $<30 \mathrm{~mL} / \mathrm{min}$ & 7 & No & Popliteal artery & Discharged \\
\hline 9 & $<30 \mathrm{~mL} / \mathrm{min} \mathrm{CVVH}$ & 4 & Hemothorax & No & Discharged \\
\hline 10 & $<30 \mathrm{~mL} / \mathrm{min}$ & 17 & No & Splanchnic vessels & Discharged \\
\hline 11 & $<30 \mathrm{~mL} / \mathrm{min} \mathrm{CVVH}$ & 6 & No & No & Discharged \\
\hline
\end{tabular}

$C V V H$, Continuous venovenous hemofiltration; $D V T$, deep vein thrombosis.

progression earlier in the sequence of reactions, the evidence for incomplete suppression of thrombin generation with direct thrombin inhibitors, the evidence for rebound hypercoagulability with thrombin inhibitors, and the clinical results with fondaparinux. ${ }^{3}$

- Patients with renal failure have an increased risk of both thrombotic and bleeding complications; however, several antithrombotic drugs undergo renal clearance. Therefore, estimation of renal function is necessary when prescribing these drugs to patients with renal dysfunction. ${ }^{4}$

- Pharmacokinetic and clinical data in patients with chronic renal impairment are limited for several anticoagulants, and adequate administration information is often absent. Lower doses and closer anticoagulation monitoring may be advisable when these agents are used in patients with chronic renal failure. We recommend that fondaparinux sodium be used with caution in patients with moderate or severe renal dysfunction and that guidelines for labora- tory monitoring and appropriate dose adjustments are urgently required to ensure the safety of the patient. The benefits of fondaparinux over enoxaparin when administered for non-ST-segment elevation acute coronary syndrome are most marked among patients with renal dysfunction and largely explained by lower rates of major bleeding with fondaparinux. ${ }^{5}$

\section{CONCLUSIONS}

On the basis of these observations in a small sample of critically ill patients after cardiac surgery, we conclude that fondaparinux provides an adjunctive tool in the armamentarium for anticoagulation in postoperative patients with HIT. Caution is advised in patients with poor renal function, especially if foreseeing invasive procedures. Anti-Xa activity monitoring is warranted in these patients, and surgery should be delayed at least 36 hours after the last administration of fondaparinux. 


\section{References}

1. Papadopoulos S, Flynn JD, Lewis DA. Fondaparinux as a treatment option for heparin-induced thrombocytopenia. Pharmacotherapy. 2007;27:921-6.

2. Warkentin TE, Greinacher A. Heparin-induced thrombocytopenia: recognition, treatment and prevention: the Seventh ACCP Conference on Antithrombotic and thrombolytic therapy. Chest. 2005;127:416.
3. Ansell J. Factor Xa or thrombin: is factor Xa a better target? J Thromb Haemost. 2007;5(Suppl 1):60-4.

4. Grand'Maison A, Charest AF, Geerts WH. Anticoagulant use in patients with chronic renal failure. Am J Cardiovasc Drugs. 2005;5:291-305.

5. Fox KA, Bassand JP, Mehta SR, et al. Oasis 5 Investigators. Influence of renal function on the efficacy and safety of fondaparinux relative to enoxaparin in non ST segment elevation acute coronary syndromes. Ann Intern Med. 2007;147:304-10. 